\title{
Psychosocial Interventions to Enhance Treatment Adherence to Lifestyle Changes in Cardiovascular Disease: A Review of the Literature 2011-2021
}

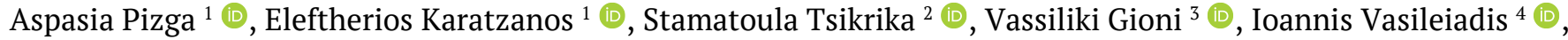 \\ Serafim Nanas ${ }^{1}$ (i), Panagiotis Kordoutis ${ }^{5 *}$ (1)
}

\footnotetext{
${ }^{1}$ Clinical Ergospirometry, Exercise and Rehabilitation Laboratory, 'Evangelismos’ General Hospital of Athens, School of M edicine, National and Kapodistrian University of Athens, Athens, GREECE

${ }^{2}$ Department of Respiratory Medicine, "Sotiria" Hospital, Athens School of Medicine, National and Kapodistrian University of Athens, Athens, GREECE

${ }^{3}$ Epistemology Field National and Kapodistrian University of Athens, Athens, GREECE

${ }^{4}$ Department of Intensive Care, 'Evangelismos' General Hospital of Athens, School of Medicine, National and Kapodistrian University of Athens, Athens, GREECE

${ }^{5}$ Social Psychology of Interpersonal Relationships, Department of Psychology, Panteion University of Social and Political Sciences, Athens, GREECE

*Corresponding Author: kordouti@panteion.gr; pkord@otenet.gr
}

Citation: Pizga, A., Karatzanos, E., Tsikrika, S., Gioni, V., Vasileiadis, I., Nanas, S. and Kordoutis, P. (2022). Psychosocial Interventions to Enhance Treatment Adherence to Lifestyle Changes in Cardiovascular Disease: A Review of the Literature 2011-2021. European Journal of Environment and Public Health, 6(1), em0102. https://doi.org/10.21601/ejeph/11582

\section{ARTICLE INFO}

Received: 6 Dec. 2021

Accepted: 14 Jan. 2022

\begin{abstract}
Patients with cardiovascular diseases face difficulty to adhere to non-pharmaceutical treatment recommendations and consequently face an increased recurrence rate, re-hospitalizations and poor quality of life. Our review of the literature over the past decade aims to be a useful tool to the enlightening of health care providers and health educators about the interventions that enhance treatment adherence to lifestyle modification. PubMed, PsycLIT, Cochrane Library, Scopus, CINAhl, PSYinfo, Web of Science, and Central databases were searched to identify articles published within the decade 2011-2021 and 42 studies met the criteria for inclusion. Our study revealed many different approaches to inconsistency in life style prescriptions focusing mainly on psychological and social factors. Interventions like increasing of knowledge, joining cardiac rehabilitation programs, development of a therapeutic alliance, behavioral techniques that reinforce self-efficacy and motivation, use of technology as reminder and creating a support network are not only effective but also lowcost programs that will play a decisive role in treatment effectiveness. Improving treatment adherence to lifestyle recommendations requires a multidimensial approach by an interdisciplinary team of health professionals. Investing in interventions that improve attitudes, beliefs, readiness and self-care, can prove to be very rewarding for patients, health and economics. The present literature review will assist health professionals and educators create experiential educational and behavioral programs that promote the adoption of healthy behavior and help maintain adherence over time. Future research is required for identifying the most effective interventions based on different lifestyles practices and cultural factors.
\end{abstract}

Keywords: treatment adherence, cardiovascular diseases, lifestyle recommendations, behavior modification, psychosocial interventions

\section{INTRODUCTION}

Cardiovascular diseases (CVDs) are the leading cause of death globally (World Health Organization, 2020) and as life expectancy increases and people live more years with cardiac disease, it is reasonable to anticipate an increased demand of patients for better quality of life. Gene mutations (Muka et al., 2016), heredity (Hanson, 2019), and psychosocial factors may be implicated in cardiac disease triggering and relapse (Everson-Rose and Lewis, 2005). Lifestyle may also play a significant role in the increased prevalence of cardiac disease seen in developed countries. Globally, smoking, physical inactivity, high blood cholesterol, high blood pressure, unhealthy diet, risky alcohol consumption, poor sleep and emotional stress are the main lifestyle risk factors for CVDs (Benjamin et al., 2019). Common therapeutic recommendations include pharmacotherapy and lifestyle risk factor modifications (Iestra et al., 2005). Patients with cardiovascular diseases often experience difficulties in following these lifestyle recommendations and this compromises treatment effectiveness with enormous consequences on health and economics (Cutler et al., 2018). Adherence to long-term therapies for chronic illnesses such us 
Table 1. Modifiable factors for non-adherence to treatment in heart patients

\begin{tabular}{ll}
\hline & Lifestyle changes recommendations \\
\hline \multirow{3}{*}{ Condition related factors } & Long term treatment (World Health Organization, 2003) \\
& Severity of the disease (Miller, 1997) \\
& Intensity of symptoms \\
& Lack of functionality (World Health Organization, 2003) \\
& Asymptomatic patients (Miller, 1997) \\
\hline \multirow{2}{*}{ Treatment-related factors } & Conflicting therapeutic recommendations/nonspecific Instructions (Carpenter et al., 2010) \\
& Complexity of treatment (Choudhry et al., 2011) \\
\hline & Lack of information/education on CVDs (Brown and Bussell, 2011) \\
& Fatigue (Kessing et al., 2016) \\
& Difficulties in memory and concentration forgetfulness (Ali et al., 2017) \\
& Shifts at work (Shan et al., 2018) \\
& Beliefs (Horne and Weinman, 1999) \\
& Reduced cost of therapies (Iuga and McGuire, 2014) \\
& Comorbidity (mental illness, stress) (Favaro et al., 2011; Gehi et al., 2005) \\
& Cognitive dissonance (Pepitone and Festinger, 1959) \\
& Social isolation and lack of support (Albus, 2010) \\
& Negative reinforcement (Byiers et al., 2014) \\
& External locus of control (Taher et al., 2015) \\
& Difficulty of access (Iuga and McGuire, 2014) \\
& Long hospital waits (Thornton et al., 2020) \\
Impersonal doctor-patient relationship (Olshansky, 2007) \\
\hline \multirow{3}{*}{ Health care factors } & Trust in the health care system (King-Shier et al., 2018) \\
\hline \multirow{2}{*}{ Cultural factors } & High in fat and calories food \\
& High frequency of social gatherings and excessive use of cars (Serour et al., 2007) \\
\hline
\end{tabular}

cardiovascular diseases in developed countries averages 50\% and the rates are even lower in developing countries (World Health Organization, 2003).

Studies indicate patients are more actively involved in their treatment when they feel that they participate and less so when they are required to passively comply to a list of guidelines (Gould and Mitty, 2010) Hence, in recent studies the term adherence is favored over the term compliance (Tilson, 2004). According to the World Health Organization, treatment adherence is the extent to which a person's behavior corresponds with the agreed recommendations-taking medication, following a diet and executing lifestyle changesfrom a healthcare provider (Sabaté and Cluster, 2001). Adherence is differentiated from passive behavior "doing as I told" and it is an active and positive behavior in which a patient and doctor work together for treatment.

Lifestyle modifications are not always a straightforward endeavor and they often undermine the already frail psychology of these patients. There are many reasons that patients have difficulties adhering to treatment (Leslie et al., 2019). According to one of the most influential theories of social psychology, the Cognitive Dissonance Theory as presented by Festinger in 1957, despite people knowing the negative impacts of an unhealthy habit as tobacco smoking, they continue to smoke. Cognitive dissonance occurs when a person holds contradictory thoughts, beliefs, or values and in order to reduce discomfort and psychological stress strive for internal psychological consistency (Pepitone \& Festinger, 1959). Even the most beneficial lifestyle modifications may instigate conflicting attitudes, trigger anxiety and discomfort, and lead people to defer or completely abandon efforts (Kiecolt, 1994).

\section{Factors Identified to Influence Non-Adherence to Lifestyle Recommendations}

Treatment resistance is multifactorial and may be related to determinants of the disease itself, including severity of symptoms and patient functionality (Miller, 1997). In addition, asymptomatic patients are more prone to underestimating the seriousness of their health condition and are less likely to adhere to recommendations for lifestyle modifications (Miller, 1997). Studies show that longer duration of treatment and more lifestyle modifications increase the likelihood for nonadherence to treatment (World Health Organization, 2003) (see Table 1). Moreover, the lack of information and education regarding the disease and the measures to improve patients' lives inhibits therapeutic adherence and bolster false assumptions about treatment (Brown \& Bussell, 2011). Specific circumstances, including fatigue (Kessing et al., 2016) memory and attention deficits (Ali et al., 2017; van der Laan et al., 2019) negative beliefs about treatment (Horne and Weinman, 1999) and external locus of health control (Taher et al., 2015), may lead patients to abandon treatment. Patients working in shifts with an irregular work schedule seem to have more difficulties following recommendations compared to patients with steady hour jobs (Shan et al., 2018).

Comorbidity also represents an important limiting factor. Patients dealing with more than one serious illnesses, taking multiple medications or with concomitant mental disorders (DiMatteo et al., 2000; Gehi et al., 2005) are less likely to adhere to treatment; for that reason, a thorough medical history before establishing a treatment plan is of paramount importance patients' diligence in following nonpharmaceutical instructions may be influenced to a significant degree by socioeconomic factors. Patients living isolated and without social support are less likely to adhere to treatment (Aggarwal et al., 2010). Lack of reinforcement $\kappa \alpha$ ı motivation for lifestyle modifications may lead to behavioral relapses 
(Byiers et al., 2014). The financial burden is another deterring factor, especially when treatment costs are not fully or at least partly covered by the insurance (Iuga and McGuire, 2014). Even patients with initially increased adherence to treatment may change their attitude over time; this may be attributed to certain impeding aspects of the healthcare system, including difficult access to examination sites (Martin et al., 2005) higher recurrent visit frequency, longer waiting times (Thornton et al., 2020) and impersonal healthcare professionals (Olshansky, 2007). Finally, cultural factors, including ethnic dietary patterns, high frequency of social gatherings (King-Shier et al., 2018) and excessive use of cars, may affect the degree of difficulty patients are facing when required to follow recommendations (Serour et al., 2007). Understanding the aforementioned factors is important for adopting managing strategies, as they indirectly contribute to worse prognosis of cardiovascular patients (Unverzagt et al., 2016), repetitive readmissions to hospital and premature death (van der Wal et al., 2010).

\section{Purpose of the Study}

We performed a review of the literature spanning the decade 2011-2021 concerning the factors favoring adherence of cardiovascular patients to recommendations for lifestyle modifications Over the past decade, many interventions have been conducted to improve treatment adherence to lifestyle modification. We collected all the studies covering the period 2011-2021 in a review in order to identify the most effective interventions and contributing to the enlightening of health practitioners. Secondarily our review can be a tool that will strengthen the heart patient's attitude towards his treatment and we hope will lead to a new way of thinking and action on treatment adherence to the long-term therapies of patients with cardiovascular diseases.

\section{METHOD}

\section{Inclusion and Exclusion Criteria}

For the present review, studies were obtained from eight electronic databases (Pubmed, PsycLIT, Cohraine Librady, Scopus, CINAhl, PSYinfo, Web of Science, and Central); we also performed a search of the grey literature in order to reduce publication bias. Inclusion criteria were studies published between 2011 and 2021, involving adults with cardiovascular disease. The 10-year time-frame was chosen in order to capture the most recent trends and to have a more manageable number of studies. Additionally, we included studies written in English, with the word "adherence" in the title or in the abstract. Although adherence and compliance terms are often used interchangeably, the latter has a more negative connotation, as it describes a passive behavior in which patients are following a list of guidelines given from the clinical practitioners. Nowadays, the term adherence is preferable, as it bears a more positive meaning and implies a collaboration between health professionals and the patient. We included articles containing the term "compliance", provided there was active patient involvement. Moreover, we included studies investigating the effectiveness of interventions emphasizing exclusively on lifestyle recommendations for improving treatment adherence. Studies attempting to identify predictors for non-adherence or for pharmaceutical adherence were not included.

\section{RESULTS}

A total of forty-two articles met the inclusion/exclusion criteria and comprised the sample for this review. The included studies included a variety of study designs: 13 observational studies, 8 correlational design, 4 quasi-experimental research, 5 randomized clinical trials, 12 systematic \& meta-analysis review. They were conducted in the following countries: Netherlands (5), Australia (2), UK (6), China (2), Germany (1), Canada (2), Egypt (1), Ethiopia (1), South Korea (2), Poland: (3), Finland (1), Jordan (4), Sweden (1), Italy (2), Pakistan (1), Brazil (2), USA (2), France (1), Spain (1), and Iran (2).

\section{Interventions to Enhance Treatment Adherence to Lifestyle Change Recommendations}

\section{Cardiac rehabilitation programs}

Cardiac rehabilitation is a multidisciplinary and individualized intervention designed to improve patients' quality of life; they include exercise, nutritional support, stress management techniques, and psychoeducation. These programs may contribute to modification of lifestyle behaviors, as participants may feel more secure exercising under specialist supervision. Participation of patients in supervised cardiac rehabilitation programs may increase adherence to treatment and contribute to positive lifestyle changes, including increased physical activity.

\section{Improving knowledge and health education}

Increased awareness may substantially enhance understanding of factors affecting health and illness and may help patients adhere to therapeutic instructions. Knowledge leads to reduced anxiety and better sense of control. In addition, patients that are actively involved in the educational process are more likely to adopt positive lifestyle changes compared to those that are passive receivers of information.

\section{Strengthen the therapeutic relationship}

Treatment alliance is an important aspect in treatment process and it can have a strong impact in health outcomes. A productive therapeutic relationship will increase treatment adherence. Cultivation a feeling of participation rather than obedience will aloud patient to feel safe and understood. Moreover, patients that are given frequent reminders from health practitioners, those that can have prompt access to a physician for an instant consult, as well as those that undergo individualized treatment have more positive feelings and trust for their physician and their treatment.

\section{Patient-tailored and behavioral interventions}

Behavioral interventions are of paramount importance for modifying patient attitudes and behaviors. Positive feedback and negative feedback elimination, as well as desensitization to reduce fears regarding treatment are examples of techniques that enhance adherence to treatment. Change of behavior requires time, gradual empowerment and motivation 
during all steps involved. Behavioral interventions are beneficial for patients with resistance to treatment; history of treatment dropouts; depression; decreased motivation; and unhealthy lifestyle habits. Reinforcement is key to successful behavior change strategies.

\section{Early detection of resistance to treatment}

A potential tool detecting patient adherence to treatment and their ability to exert self-control will greatly assist in achieving individualized management. The Theory of Planned Behavior (TPB) is a social cognitive model that predicts an individual's intention to engage in a behavior at a specific time and place. TPB has been used successfully to predict treatment adherence in chronic illness and therefore is an important tool for health care providers in cardiovascular diseases (Mckenna, 1996; Rich et al., 2015). Moreover, timely detection of mental disorders may contribute to developing an individualized therapeutic scheme that takes into account the mental and physical needs of each patient.

\section{Beliefs, attitudes, and internal locus of control}

People believing that their behavior greatly determines their health status are more likely to change their lifestyle in order to improve their health. On the other hand, those believing that their health is mostly determined by unpredictable factors rather than behavior are less probable to proceed in major lifestyle modifications. Health practitioners should encourage patients to embrace a more responsible outlook on their health.

\section{Stress management techniques}

Psychological support, with interventions including emotion management, incentivization, realistic goal setting, can positively affect mental and physical status of cardiovascular patients. Replacing harmful habits with healthier ones is extremely important for treatment success. The psychologist plays the role of the patient's partner, detecting and helping them deal with their fears, anxieties and other obstacles encountered during the process of changing lifestyle. Relaxation techniques help reducing stress and anxiety regarding disease, as stress may lead to emergence and persistence of unhealthy behaviors such as consuming junk food, smoking and inactivity. Stress management may contribute to therapeutic adherence and prevention of recurrences.

\section{Enhancement self-care and self-efficacy}

Self- efficacy seems to be a predictor for treatment adherence and interventions that enhance self - efficacy could play an important role in disease management and self-care behaviors.

\section{Family and community support}

The support provided by family members and friends is very important to the patient. Involving friends and family in treatment may improve patient's perspective and assist in practical issues, as encouraging and supporting exercise. In fact, in order to support a patient, friends and family members frequently adopt positive changes in their lifestyle as well, sowing the seeds of good health.

\section{Use of technology for monitoring, organizing, motivation, and reminding}

The use of a reminder device may assist adherence to both treatment and lifestyle changes. Smartphone applications reminding and encouraging exercise, diet control and relaxation techniques have been proven helpful for patients with memory deficits or with lack of motivation. Tailored tele monitoring was found also effective to educate patients with $\mathrm{HF}$ and to improve their self-care abilities and sense of selfefficacy.

\section{Regular outpatient contact, follow up and visits}

Frequent communication using phone calls, texting, and healthcare provider visits is positively viewed by patients and may help them maintain focus on their goals and adhere to the treatment plan.

Table 2 and Table 3 summarizes our review in this study.

Table 2. Characteristics of studies that integrated treatment adherence for lifestyle recommendations included in this review

\begin{tabular}{|c|c|c|c|c|c|}
\hline Lead author & Year & Journal of publication & Type of study & Population & Factors enhance adherence \\
\hline Sol et al. & 2011 & $\begin{array}{c}\text { European Journal of } \\
\text { Cardiovascular Nursing }\end{array}$ & $\begin{array}{l}\text { Observational cohort } \\
\text { study }\end{array}$ & $\begin{array}{l}25 \text { patients with } \\
\text { cerebrovascular } \\
\text { disease, } \mathrm{CHD} \text {, or } \\
\text { peripheral arterial } \\
\text { disease }\end{array}$ & $\begin{array}{l}\text { Self-efficacy improved adherence to physical } \\
\text { activity and food choices }\end{array}$ \\
\hline
\end{tabular}

\begin{tabular}{lcccc}
\hline Heydari et al. 2011 & $\begin{array}{c}\text { Journal of Cardiovascular } \\
\text { Nursing }\end{array}$ & $\begin{array}{c}\text { Descriptive } \\
\text { correlational design }\end{array}$ & 108 HF patients & $\begin{array}{c}\text { Patients perceive their therapeutic regimen as } \\
\text { a challenge }\end{array}$ \\
\hline
\end{tabular}

Using self-management interventions can be

helpful in adhering to medication regimen and changing lifestyle in choosing low fat diet. Baljani et al. $2012 \begin{gathered}\text { Avicenna Journal of } \\ \text { Nursing \& Midwifery Care }\end{gathered} \begin{gathered}\text { Quasi-experimental } \\ \text { research }\end{gathered} \begin{gathered}92 \text { patients with } \\ \text { cardiovascular disease }\end{gathered} \begin{gathered}\text { In patients with cardiovascular diseases whom } \\ \text { specialists have desired long-term lifestyle }\end{gathered}$ changes, self-management is in effective measure that increases their control over their condition.

\begin{tabular}{lccccc}
\hline $\begin{array}{l}\text { Martin and } \\
\text { Woods }\end{array}$ & 2012 & $\begin{array}{c}\text { Journal of Aging and } \\
\text { Physical Activity }\end{array}$ & Correlational study & $\begin{array}{c}24 \text { long-term adherers } \\
\text { after cardiac event }\end{array}$ & $\begin{array}{c}\text { Belief in health benefits, structure class } \\
\text { emotional social support } \\
\text { self-efficacy }\end{array}$ \\
\hline Guiraud et al. 2012 & $\begin{array}{c}\text { Archives of Physical } \\
\text { Medicine and } \\
\text { Rehabilitation }\end{array}$ & $\begin{array}{c}\text { Prospective and } \\
\text { randomized study }\end{array}$ & 29 patients & Telephone support based on accelerometer \\
\hline
\end{tabular}


Table 2. Characteristics of studies that integrated treatment adherence for lifestyle recommendations included in this review

\begin{tabular}{|c|c|c|c|c|c|}
\hline Lead author & Year & Journal of publication & Type of study & Population & Factors enhance adherence \\
\hline
\end{tabular}

Marti et al. 2013 Congestive Heart Failure $\quad \begin{aligned} & \text { Prospective } \\ & \text { cohort study }\end{aligned}$

Self-care education

Lower resource utilization and better health status

\begin{tabular}{|c|c|c|c|c|c|}
\hline $\begin{array}{l}\text { Nieuwenhuis et } \\
\text { al. }\end{array}$ & 2012 & Elsevier & $\begin{array}{l}\text { Descriptive, } \\
\text { prospective design }\end{array}$ & $\begin{array}{c}648 \text { patients with HF } \\
\text { used data from the } \\
\text { COACH study } \\
\text { coordinating study } \\
\text { evaluating outcomes of } \\
\text { advising and } \\
\text { counseling in heart } \\
\text { failure }\end{array}$ & $\begin{array}{l}\text { Knowledge on HF and educational support } \\
\text { positively affected compliance with weighing } \\
\text { and fluid restriction but not exercise }\end{array}$ \\
\hline Griffo et al. & 2013 & $\begin{array}{c}\text { International Journal of } \\
\text { Cardiology }\end{array}$ & $\begin{array}{l}\text { Prospective, } \\
\text { longitudinal, } \\
\text { multicenter registry } \\
\text { with on-line web- } \\
\text { based data collection }\end{array}$ & $\begin{array}{l}\text { 1,262 patients after } \\
\text { coronary artery bypass } \\
\text { grafting CABG or PCI }\end{array}$ & $\begin{array}{c}\text { Effective secondary prevention through cardiac } \\
\text { rehabilitation }\end{array}$ \\
\hline $\begin{array}{l}\text { Tawalbeh and } \\
\text { Ahmad }\end{array}$ & 2014 & Clinical Nursing Research & $\begin{array}{c}\text { Group pretest- } \\
\text { posttest study design }\end{array}$ & 153 CAD patients & Cardiac educational program \\
\hline
\end{tabular}
Eshah et al. 2013 Nursing \& Health Sciences $\begin{gathered}\text { Quasi-experimental } \\ \text { research }\end{gathered}$

104 ACS

Predischarge education participants from the experimental group scored significantly higher

on three components of healthy lifestyle, health responsibility, nutrition, and interpersonal relations

\begin{tabular}{|c|c|c|c|c|c|}
\hline Maeda et al. & 2013 & $\begin{array}{l}\text { International Journal of } \\
\text { Behavioral Medicine }\end{array}$ & Correlational study & $\begin{array}{l}252 \text { chronic HF } \\
\text { patients }\end{array}$ & $\begin{array}{c}\text { Self-efficacy mediated the associations of } \\
\text { social support and depression with treatment } \\
\text { adherence }\end{array}$ \\
\hline Janssen et al. & 2013 & $\begin{array}{l}\text { Journal of Behavioral } \\
\text { Medicine }\end{array}$ & Correlational study & $\begin{array}{l}210 \text { post cardiac } \\
\text { rehabilitation patients }\end{array}$ & $\begin{array}{c}\text { Self-regulation lifestyle program effects on } \\
\text { exercise levels at } 6 \text { months post cardiac } \\
\text { rehabilitation }\end{array}$ \\
\hline $\begin{array}{l}\text { Slovinec } \\
\text { D’Angelo et al. }\end{array}$ & 2014 & Health Psychology & $\begin{array}{c}\text { Prospective, } \\
\text { longitudinal design }\end{array}$ & $\begin{array}{c}801 \text { patients } \\
\text { hospitalized for CHD }\end{array}$ & $\begin{array}{c}\text { Autonomous motivation } \\
\text { Self-efficacy in adherence to exercise }\end{array}$ \\
\hline Tawalbeh et al. & 2017 & Clinical Nursing Research & Correlational study & 84 patients with CAD & Cardiac educational program \\
\hline $\begin{array}{l}\text { Piotrowicz et } \\
\text { al. }\end{array}$ & 2015 & $\begin{array}{c}\text { European Journal of } \\
\text { Preventive Cardiology }\end{array}$ & $\begin{array}{l}\text { Randomized ( } 2: 1) \\
\text { controlled trial }\end{array}$ & 111 HF patients & Home-based tele monitored NW \\
\hline Boyne et al. & 2014 & $\begin{array}{c}\text { European Journal of } \\
\text { Cardiovascular Nursing }\end{array}$ & $\begin{array}{l}\text { Randomized } \\
\text { controlled trial }\end{array}$ & 382 patients with $\mathrm{HF}$ & Tailored tele monitoring \\
\hline Silva et al. & 2015 & $\begin{array}{l}\text { Revista Latino-Americana } \\
\text { de Enfermagem }\end{array}$ & $\begin{array}{l}\text { Cross-sectional } \\
\text { study }\end{array}$ & 340 HF patients & $\begin{array}{l}\text { Live with family } \\
\text { Three or more previous nursing appointments }\end{array}$ \\
\hline Urbinati et al. & 2015 & $\begin{array}{c}\text { European Journal of } \\
\text { Preventive Cardiology }\end{array}$ & Correlational study & $\begin{array}{l}\text { 11,706 patients with } \\
\text { AMI }\end{array}$ & $\begin{array}{c}\text { Post-discharge cardiac visit } \\
\text { referral to cardiac rehabilitation }\end{array}$ \\
\hline Ok and Choi & 2015 & $\begin{array}{l}\text { Korean Journal of Adult } \\
\text { Nursing }\end{array}$ & $\begin{array}{l}\text { Correlational } \\
\text { research }\end{array}$ & $\begin{array}{l}280 \text { outpatients with } \\
\text { heart failure }\end{array}$ & $\begin{array}{l}\text { Lower functional status } \\
\text { social support, } \\
\text { greater knowledge of heart failure }\end{array}$ \\
\hline Johnston et al. & 2016 & American Heart Journal & $\begin{array}{l}\text { Multicenter, } \\
\text { randomized trial }\end{array}$ & $\begin{array}{l}174 \text { ticagrelor-treated } \\
\text { MI patients }\end{array}$ & Use of an interactive patient support tool \\
\hline $\begin{array}{l}\text { Akhu-Zaheya } \\
\text { and Shiyab }\end{array}$ & 2017 & $\begin{array}{l}\text { International Journal of } \\
\text { Medical Informatics }\end{array}$ & $\begin{array}{l}\text { Randomized } \\
\text { controlled trial }\end{array}$ & 160 patients & $\begin{array}{l}\text { It is documented that SMS is effective in } \\
\text { improving adherence to a healthy diet and } \\
\text { medication. } \\
\text { SMS could be a promising solution for } \\
\text { management of different chronic diseases }\end{array}$ \\
\hline $\begin{array}{l}\text { Kähkönen et } \\
\text { al. }\end{array}$ & 2018 & $\begin{array}{l}\text { International Committee } \\
\text { of Medical Journal Editors }\end{array}$ & $\begin{array}{l}\text { Analytical multi- } \\
\text { hospital survey } \\
\text { study }\end{array}$ & $\begin{array}{l}416 \text { post-percutaneous } \\
\text { coronary intervention } \\
\text { patients }\end{array}$ & $\begin{array}{c}\text { Male gender, } \\
\text { close personal relationship, } \\
\text { longer education, and lower LDL-cholesterol, } \\
\text { Longer duration of coronary heart disease } \\
\text { without previous percutaneous coronary } \\
\text { intervention }\end{array}$ \\
\hline $\begin{array}{l}\text { Jankowska- } \\
\text { Polańska et al. }\end{array}$ & 2017 & $\begin{array}{l}\text { Journal of Education, } \\
\text { Health and Sport. }\end{array}$ & $\begin{array}{l}\text { Cross sectional } \\
\text { study }\end{array}$ & $170 \mathrm{HF}$ patients & $\begin{array}{c}\text { Cognitive function is an independent } \\
\text { contributor. } \\
\text { Being in a relationship } \\
\text { education are independent predictors of better } \\
\text { compliance. }\end{array}$ \\
\hline
\end{tabular}


Table 2 (Continued). Characteristics of studies that integrated treatment adherence for lifestyle recommendations included in this review

\begin{tabular}{lccccc}
\hline Lead author & Year & Journal of publication & Type of study & Population & Factors enhance adherence \\
\hline Kosobucka et al. & 2018 & Dovepress & $\begin{array}{c}\text { Observational cohort } \\
\text { clinical study }\end{array}$ & 221 patients with ACDS & Age previous MI \\
\hline Santo et al. & 2018 & $\begin{array}{c}\text { International Journal of } \\
\text { Behavioral Nutrition and } \\
\text { Physical Activity }\end{array}$ & $\begin{array}{c}\text { Randomized clinical } \\
\text { trial }\end{array}$ & $\begin{array}{c}705 \text { patients with } \\
\text { coronary heart disease }\end{array}$ & Lifestyle-focused text-message program \\
\hline Lee et al. & 2018 & $\begin{array}{c}\text { Health Quality Life } \\
\text { Outcomes }\end{array}$ & Cross-sectional study & $\begin{array}{c}417 \text { post-AMI patients } \\
\text { with acute myocardial } \\
\text { infarction }\end{array}$ & $\begin{array}{c}\text { Early detection of patients with poor } \\
\text { adherence to medication }\end{array}$ \\
\hline Seid et al. & 2019 & PLoS One & Cross-sectional study & $\begin{array}{c}310 \text { adult heart failure } \\
\text { patients }\end{array}$ & $\begin{array}{c}\text { No chronic comorbidity, } \\
\text { Good level of heart failure, } \\
\text { Knowledge }\end{array}$ \\
\hline $\begin{array}{l}\text { Metwaly and } \\
\text { Zatton }\end{array}$ & 2020 & $\begin{array}{c}\text { Egyptian Journal of } \\
\text { Health Care }\end{array}$ & $\begin{array}{c}\text { Pre/posttest quasi- } \\
\text { experimental design }\end{array}$ & 35 adult patients & Health educational program \\
\hline Chew et al. & 2021 & $\begin{array}{c}\text { International Journal of } \\
\text { Nursing Studies }\end{array}$ & $\begin{array}{c}\text { A two-arm randomized } \\
\text { controlled trial }\end{array}$ & $\begin{array}{c}144 \text { patients with heart } \\
\text { failure }\end{array}$ & Self-care intervention \\
\hline Suhail at al. & 2021 & $\begin{array}{c}\text { Health Quality Life } \\
\text { Outcomes }\end{array}$ & $\begin{array}{c}\text { Cross-sectional study } \\
251 \text { patients with IHD }\end{array}$ & Adequate health literacy \\
\hline
\end{tabular}

Table 3. Reviews, systematic reviews, \& meta-analyses that integrated treatment adherence for lifestyle recommendations included in this review

\begin{tabular}{|c|c|c|c|c|c|}
\hline Lead author & Year & Journal of publication & Type of study & $\begin{array}{c}\text { Number of } \\
\text { subjects/studies }\end{array}$ & Factors enhance adherence \\
\hline Tierney et al. & 2012 & Heart Failure Reviews & $\begin{array}{l}\text { A systematic review of } \\
\text { controlled studies }\end{array}$ & 3,231 patients & $\begin{array}{c}\text { Exercise prescriptions } \\
\text { Goal setting } \\
\text { Feedback } \\
\text { Problem-solving } \\
\text { Self-efficacy } \\
\end{array}$ \\
\hline Murray et al. & 2012 & $\begin{array}{l}\text { BMC Cardiovascular } \\
\text { Disorders }\end{array}$ & $\begin{array}{l}\text { A systematic narrative } \\
\text { review of quantitative } \\
\text { observational studies }\end{array}$ & $\begin{array}{l}32 \text { studies } \\
\text { (374 factors) }\end{array}$ & $\begin{array}{l}\text { Support from family \& friends } \\
\text { Transport \& other costs } \\
\text { Beliefs about the causes of illness \& } \\
\text { lifestyle change }\end{array}$ \\
\hline $\begin{array}{l}\text { Oosterom-Calo } \\
\text { et al. }\end{array}$ & 2012 & Heart Failure Review & $\begin{array}{l}\text { A systematic literature } \\
\text { review \& meta- } \\
\text { analysis }\end{array}$ & 569 studies & Institutionalized patients in the past \\
\hline $\begin{array}{l}\text { Pfaeffli Dale et } \\
\text { al. }\end{array}$ & 2016 & $\begin{array}{l}\text { European Journal of } \\
\text { Preventive Cardiology }\end{array}$ & $\begin{array}{l}\text { A comprehensive } \\
\text { literature search }\end{array}$ & 7 studies & $\begin{array}{l}3 \text { studies were effective at improving } \\
\text { adherence to medication. } \\
2 \text { studies increased physical activity } \\
\text { behavior. Simple text messaging } \\
\text { interventions appeared to be most } \\
\text { effective. }\end{array}$ \\
\hline Unverzagt et al. & 2016 & $\begin{array}{l}\text { Deutsches Ärzteblatt } \\
\text { International }\end{array}$ & Systematic review & $\begin{array}{c}42 \text { trials } \\
\text { (211 full text articles) }\end{array}$ & $\begin{array}{c}\text { Patients' assuming responsibility for } \\
\text { their own health } \\
\text { Patient education } \\
\text { Regular follow-up contacts }\end{array}$ \\
\hline Leung et al. & 2017 & $\begin{array}{l}\text { International Journal of } \\
\text { Environmental Research } \\
\text { and Public Health }\end{array}$ & Literature review & 19 studies & $\begin{array}{l}\text { Attrition is the most common indicator } \\
\text { used, followed by attendance, self- } \\
\text { monitoring, and dietary adherence. } \\
\text { Factors that may predict better } \\
\text { adherence were being in action or } \\
\text { maintenance stage of change, older age, } \\
\text { higher education, healthier eating, and } \\
\text { PA behavior at baseline and more initial } \\
\text { weight loss. }\end{array}$ \\
\hline $\begin{array}{l}\text { Stonerock and } \\
\text { Blumenthal } \\
\end{array}$ & 2017 & $\begin{array}{c}\text { Progress in } \\
\text { Cardiovascular Diseases } \\
\end{array}$ & Review & - & $\begin{array}{c}\text { Motivational interviewing } \\
\text { Trans theoretical model approaches }\end{array}$ \\
\hline $\begin{array}{l}\text { Kassavou and } \\
\text { Sutton }\end{array}$ & 2018 & $\begin{array}{l}\text { Health Psychology } \\
\text { Review }\end{array}$ & Meta-analysis & $\begin{array}{c}17 \text { randomized } \\
\text { controlled trials }(38,671 \\
\text { participants }) \\
\end{array}$ & $\begin{array}{l}\text { Behavioral change techniques (BCTs) } \\
\text { 'tailored' and 'information about health } \\
\text { consequences' }\end{array}$ \\
\hline Debon et al. & 2019 & $\begin{array}{l}\text { Diabetes \& Metabolic } \\
\text { Syndrome: Clinical } \\
\text { Research \& Reviews }\end{array}$ & Systematic review & 24 studies & $\begin{array}{l}\text { Mobile health applications and self- } \\
\text { monitoring }\end{array}$ \\
\hline
\end{tabular}


Table 3 (continued). Reviews, systematic reviews, \& meta-analyses that integrated treatment adherence for lifestyle recommendations included in this review

\begin{tabular}{|c|c|c|c|c|c|}
\hline Lead author & Year & Journal of publication & Type of study & $\begin{array}{c}\text { Number of } \\
\text { subjects/studies }\end{array}$ & Factors enhance adherence \\
\hline Rashidi et al. & 2020 & $\begin{array}{l}\text { International Journal of } \\
\text { Nursing Studies }\end{array}$ & $\begin{array}{l}\text { Systematic review \& } \\
\text { qualitative synthesis }\end{array}$ & 22 articles & $\begin{array}{c}\text { Exercise } \\
\text { Support } \\
\text { Mentorship }\end{array}$ \\
\hline Westland et al. & 2020 & European Heart Journal & Systematic review & $\begin{array}{c}166 \text { full-text articles on } \\
\text { CAD studies }\end{array}$ & Self-care interventions \\
\hline Akinosun et al. & 2021 & JMIR Mhealth Uhealth & $\begin{array}{l}\text { Systematic review \& } \\
\text { meta-analysis }\end{array}$ & & $\begin{array}{l}\text { Cell phones, smartphones, personal } \\
\text { computers, and wearables coupled with } \\
\text { technologies such as the Internet, SMS, } \\
\text { software applications, and mobile } \\
\text { sensors } \\
\text { Digital interventions }\end{array}$ \\
\hline
\end{tabular}

\section{DISCUSSION}

Understanding the circumstances and context that lead to unhealthy behaviors is challenging. Healthcare professionals are required to collaborate with each other, as well as with patients and their friends and family in order to develop and implement a treatment plan tailored to each patient's specific needs. During the past decade, numerous studies were conducted looking into the effectiveness of interventions aimed to increase adherence of cardiovascular patients to lifestyle modifications. Their results underscore the value of establishing a treatment bond between the physician and the patient. A good therapeutic rapport is predictive of adherence to treatment and it is enhanced through trust, an individualized approach and the potential for instant consult in case of emergency. Patients who feel that they are being heard are also good listeners. Various interventions, including enrolling patients in cardiac rehabilitation programs, may be greatly beneficial, as they incorporate supervision from a multidisciplinary team and enhanced guidance. Moreover, a patient-tailored approach with behavioral interventions promotes a successful therapeutic adherence. Several studies have also proven the importance of early recognition of resistance to treatment, mainly in patients with a history of unhealthy habits, previous failed attempts to change, living alone or facing additional health issues. Patient readiness to change and to adopt new habits has not been adequately investigated, although it seems to affect adherence prognosis (Mckenna, 1996; Rich et al., 2015). Transtheoretical model (TM) is an integrative theory of therapy that assesses an individual's readiness to act on a new healthier behavior, and provides strategies, or processes of change to guide the individual (Prochaska \& DiClemente, 2005). TM posits that individuals move through six stages of change: precontemplation, contemplation, preparation, action, maintenance, and termination. The implementation of this model for detecting readiness to change, especially in patients prone to treatment dropout, as well as for the support of this changing process step-by-step, may offer a valuable tool to health educators.

Additionally, providing information through educational programs for patients and family members may set aside false beliefs and reestablish the control people have over their health, acknowledging that their behavior contributes both to health and disease. According to the social cognitive theory of health belief model (HBM), developed in order to understand failure to adopt disease prevention strategies, people will respond with positive changes if they recognize and understand that poor health may be avoided or prevented, and if they believe that they are able to adopt a new and beneficial behavior (Janz and Becker, 1984). Therefore, patient education and awareness campaigns will help increase understanding of therapeutic recommendations, as a prerequisite for improving health. Increased awareness regarding the disease and the way behavior affects health is important but not sufficient for change per se. Frequently, patients struggle to give up harmful habits despite being aware of their adverse impact or neglect to exercise despite knowing the benefits. Patients often require something more than plain instructions; instead, they need experiential education on new behavioral patterns before changing their lifestyle (Kolb and Kolb, 2009; O’Grady et al., 2008). A step-by-step educational process implemented by experts on how to abstain from a negative habit and how to replace it with a more beneficial one is of utmost importance. Educational interventions focused on increasing self-efficacy and self-care have been proven very effective in enhancing self-confidence, preserving functionality and maintaining patients' mental health. Regular reminders, phone calls, text messages and notifications indicate interest and greatly contribute to maintaining a good therapeutic attitude over time, especially in the elderly and people with memory issues.

Patients receiving psychotherapy (Wallert et al., 2018) or those using stress management techniques (Yu et al., 2010) are less likely to regress to previous behaviors regarding adherence to recommendations, and exhibit less distress and anxiety. Family members and friends play a very important role in the course of the patient's adherence to treatment; that said, people living alone or lacking a supportive network should be encouraged to seek help from organizations supporting patients with chronic diseases. Group therapies are highly beneficial for patients that struggle to discontinue unhealthy habits and need support by people experiencing similar feelings. Promoting feelings of social acceptance and providing support by organizations and family members significantly improve the emotional status of patients.

Despite the interesting findings regarding factors affecting adherence to treatment, studies included in this review have certain limitations. Measurement of adherence to treatment is typically performed using self-administered questionnaires; therefore, these studies are prone to social desirability bias. In 
addition, most studies do not take into account cultural factors that may affect adherence; participant characteristics also exhibit great heterogeneity.

As the aim of the present review is to identify interventions facilitating adherence to treatment and not to list factors predicting non-adherence, there is a risk for selection bias. Finally, there are other studies with the same objectives that were not included, as they did not exclusively focus on patients with cardiovascular disease, but involved various chronic conditions. Many interventions effectively increase adherence to treatment for a specific group of patients may be beneficial for people with a different chronic disease; this warrants further investigation.

Future studies should focus on the effectiveness of various psychosocial models for behavioral change, in order to further analyze processes contributing to readiness for change and long-term maintenance of this behavior change. Furthermore, as the number of younger patients suffering from cardiovascular disease has risen in the recent years, it is important for future studies to identify variations in factors affecting adherence in different age groups. Finally, attention should be drawn in developing and administering more objective outcome measures for adherence to nonpharmaceutical recommendations.

In conclusion, health policy makers should take into account factors affecting change of behavior and invest in studying and developing flexible and cost-effective interventions encouraging adoption and long-term maintenance of a healthy lifestyle.

\section{IMPLICATIONS IN ENVIROMENTAL AND PUBLIC HEALTH}

The lack of adherence to non-pharmaceutical recommendations is a serious issue for cardiovascular patients worldwide, leading to hospitalizations, poor quality of life, and increased risk for clinical events and death. In addition, it undermines the effectiveness of health care systems and increases annual costs. The literature indicates that all these concerns may be avoided by investing in cost-effective interventions that promote patient adherence to treatment. The recognition of factors affecting lifestyle modifications of cardiovascular patients increases the chances of adopting positive health behaviors and has extended applications for the health education field. Health professionals and educators can contribute to treatment adherence in the following ways:

1. design of educational programs based on experiential learning for health professionals,

2. compassion cultivation training programs for health providers and for the patient's relatives,

3. inclusion of theoretical models of behavior change from social sciences in the training of specialists and patients (Educational programs should focus on influence attitudes, beliefs, and in the timely acceptance of disease and its preparation for change),

4. working with social health agencies to support the vulnerable population,
5. promotion of enhanced cardiac rehabilitation programs,

6. patient education in the use of technologies, apps as motivation reminders, and organize treatment goals,

7. promote collective actions and awareness strategies in cardiovascular health,

8. creating campaigns that promote the adoption of a healthy lifestyle behaviors with inspiring role models,

9. collaboration with experts to study and evaluation of the reported actions, and

10. conduct research related to patients' satisfaction from his therapy, health practitioners, and support service providers.

We recommend health professionals use our findings to enhance therapeutic process with effective interventions and help create a flexible treatment framework where the patient receives individualized support at all stages of the change.

Author contributions: AP and LK: contributed to the conception and design of the study. All authors contributed to the acquisition, analysis, and interpretation of data; drafting the article; and revising it critically for important academic content. All authors approved the final version for publication. All authors agree with the results and conclusions.

Funding: No external funding is received for this article.

Declaration of interest: The authors declare that they have no competing interests.

Ethics approval and consent to participate: Not applicable.

Availability of data and materials: All data generated or analyzed during this study are available for sharing when appropriate request is directed to corresponding author.

\section{REFERENCES}

Aggarwal, B., Liao, M., Allegrante, J. P. and Mosca, L. (2010). Low social support level is associated with non-adherence to diet at 1 year in the family intervention trial for heart health (FIT heart). Journal of Nutrition Education and Behavior, 42(6), 380-388. https://doi.org/10.1016/j.jneb. 2009.08.006

Akhu-Zaheya, L. M. and Shiyab, W. Y. (2017). The effect of short message system (SMS) reminder on adherence to a healthy diet, medication, and cessation of smoking among adult patients with cardiovascular diseases. International Journal of Medical Informatics, 98, 65-75. https://doi.org/ 10.1016/j.ijmedinf.2016.12.003

Akinosun, A. S., Polson, R., Diaz-Skeete, Y., De Kock, J. H., Carragher, L., Leslie, S., Grindle, M. and Gorely, T. (2021). Digital technology interventions for risk factor modification in patients with cardiovascular disease: Systematic review and meta-analysis. JMIR mHealth and uHealth, 9(3), e21061. https://doi.org/10.2196/21061

Albus, C. (2010). Psychological and social factors in coronary heart disease. Annals of Medicine, 42(7), 487-494. https://doi.org/10.3109/07853890.2010.515605 
Ali, M. A., Yasir, J., Sherwani, R. N., Fareed, M., Arshad, F., Abid, F. and Fatima, K. (2017). Frequency and predictors of non-adherence to lifestyle modifications and medications after coronary artery bypass grafting: A cross-sectional study. Indian Heart Journal, 69(4), 469-473. https://doi.org/ 10.1016/j.ihj.2017.05.017

Baljani, E., Salimi, S., Rahimi, J., Amanpour, E., Parkhashjou, M., Sharifnejad, A. and Poyan, S. (2012). The effect of education on promoting self efficacy in patients with cardiovascular disease. Journal of Kermanshah University of Medical Sciences, 16(3), e78799.

Benjamin, E. J., Muntner, P., Alonso, A., Bittencourt, M. S., et al. (2019). Heart disease and stroke statistics-2019 update: A report from the American Heart Association. Circulation, 139(10), e56-e528. https://doi.org/10.1161/CIR.000000000 0000746

Boyne, J. J., Vrijhoef, H. J., Spreeuwenberg, M., De Weerd, G., Kragten, J. and Gorgels, A. P. (2014). Effects of tailored tele monitoring on heart failure patients' knowledge, self-care, self-efficacy and adherence: A randomized controlled trial. European Journal of Cardiovascular Nursing, 13(3), 243-252. https://doi.org/10.1177/1474515113487464

Brown, M. T. and Bussell, J. K. (2011). Medication adherence: WHO cares? Mayo Clinic Proceedings, 86(4), 304-314. https://doi.org/10.4065/mcp.2010.0575

Byiers, B., Dimian, A., McComas, J. J. and Symons, F. J. (2014). Effects of positive and negative reinforcement in a concurrent operants arrangement on compliance and problem behavior. Acta de Investigación Psicológica, 4(3), 1758-1772. https://doi.org/10.1016/S2007-4719(14)709780

Carpenter, D. M., DeVellis, R. F., Fisher, E. B., DeVellis, B. M., Hogan, S. L. and Jordan, J. M. (2010). The effect of conflicting medication information and physician support on medication adherence for chronically ill patients. Patient Education and Counseling, 81(2), 169-176. https://doi.org/10.1016/j.pec.2009.11.006

Chew, H. S. J., Sim, K. L. D., Choi, K. C. and Chair, S. Y. (2021). Effectiveness of a nurse-led temporal self-regulation theory-based program on heart failure self-care: A randomized controlled trial. International Journal of Nursing Studies, 115, 103872. https://doi.org/10.1016/ j.ijnurstu.2021.103872

Choudhry, N. K., Fischer, M. A., Avorn, J., Liberman, J. N., Schneeweiss, S., Pakes, J., Brennan, T. A. and Shrank, W. H. (2011). The implications of therapeutic complexity on adherence to cardiovascular medications. Archives of Internal Medicine, 171(9), 814-822. https://doi.org/10.1001/ archinternmed.2010.495

Cutler, R. L., Fernandez-Llimos, F., Frommer, M., Benrimoj, C. and Garcia-Cardenas, V. (2018). Economic impact of medication non-adherence by disease groups: A systematic review. BMJ Open, 8(1), e016982. https://doi.org/10.1136/ bmjopen-2017-016982 da Silva, A. F., Cavalcanti, A. C. D., Malta, M., Arruda, C. S., Gandin, T., da Fé, A. and Rabelo-Silva, E. R. (2015). Treatment adherence in heart failure patients followed up by nurses in two specialized clinics. Revista LatinoAmericana de Enfermagem [Latin American Journal of Nursing], 23(5), 888-894. https://doi.org/10.1590/01041169.0268.2628

Debon, R., Coleone, J. D., Bellei, E. A. and De Marchi, A. C. B. (2019). Mobile health applications for chronic diseases: A systematic review of features for lifestyle improvement. Diabetes and Metabolic Syndrome, 13(4), 2507-2512. https://doi.org/10.1016/j.dsx.2019.07.016

DiMatteo, M. R., Lepper, H. S. and Croghan, T. W. (2000). Depression is a risk factor for noncompliance with medical treatment: Meta-analysis of the effects of anxiety and depression on patient adherence. Archives of Internal Medicine, 160(14), 2101-2107. https://doi.org/10.1001/ archinte.160.14.2101

Eshah, N. F. (2013). Predischarge education improves adherence to a healthy lifestyle among Jordanian patients with acute coronary syndrome. Nursing and Health Sciences, 15(3), 273-279.https://doi.org/10.1111/nhs.12018

Everson-Rose, S. A. and Lewis, T. T. (2005). Psychosocial factors and cardiovascular diseases. Annual Review of Public Health, 26, 469-500. https://doi.org/10.1146/annurev. publhealth.26.021304.144542

Favaro, A., Gerosa, G., Caforio, A. L., Volpe, B., Rupolo, G., Zarneri, D. and Santonastaso, P. (2011). Posttraumatic stress disorder and depression in heart transplantation recipients: The relationship with outcome and adherence to medical treatment. General Hospital Psychiatry, 33(1), 17. https://doi.org/10.1016/j.genhosppsych.2010.10.001

Gehi, A., Haas, D., Pipkin, S. and Whooley, M. A. (2005). Depression and medication adherence in outpatients with coronary heart disease: Findings from the heart and soul study. Archives of Internal Medicine, 165(21), 2508-2513. https://doi.org/10.1001/archinte.165.21.2508

Gould, E. and Mitty, E. (2010). Medication adherence is a partnership, medication compliance is not. Geriatric Nursing, 31(4), 290-298. https://doi.org/10.1016/ j.gerinurse.2010.05.004

Griffo, R., Ambrosetti, M., Tramarin, R., Fattirolli, F., Temporelli, P. L., Vestri, A. R. and ICAROS investigators. (2013). Effective secondary prevention through cardiac rehabilitation after coronary revascularization and predictors of poor adherence to lifestyle modification and medication. Results of the ICAROS survey. International Journal of Cardiology, 167(4), 1390-1395. https://doi.org/ 10.1016/j.ijcard.2012.04.069

Guiraud, T., Granger, R., Gremeaux, V., Bousquet, M., Richard, L., Soukarié, L. and Pathak, A. (2012). Telephone support oriented by accelerometric measurements enhances adherence to physical activity recommendations in noncompliant patients after a cardiac rehabilitation program. Archives of Physical Medicine and Rehabilitation, 93(12), 2141-2147. https://doi.org/10.1016/j.apmr.2012. 06.027 
Hanson, M. (2019). The inheritance of cardiovascular disease risk. Acta Paediatrica, 108(10), 1747-1756. https://doi.org/ 10.1111/apa.14813

Heydari, A., Ahrari, S. and Vaghee, S. (2011). The relationship between self-concept and adherence to therapeutic regimens in patients with heart failure. Journal of Cardiovascular Nursing, 26(6), 475-480. https://doi.org/ 10.1097/JCN.0b013e318215bb78

Horne, R. and Weinman, J. (1999). Patients' beliefs about prescribed medicines and their role in adherence to treatment in chronic physical illness. Journal of Psychosomatic Research, 47(6), 555-567. https://doi.org/ 10.1016/s0022-3999(99)00057-4

Iestra, J. A., Kromhout, D., van der Schouw, Y. T., Grobbee, D. E., Boshuizen, H. C. and van Staveren, W. A. (2005). Effect size estimates of lifestyle and dietary changes on all-cause mortality in coronary artery disease patients: A systematic review. Circulation, 112(6), 924-934. https://doi.org/ 10.1161/CIRCULATIONAHA.104.503995

Iuga, A. O. and McGuire, M. J. (2014). Adherence and health care costs. Risk Management and Healthcare Policy, 7, 3544. https://doi.org/10.2147/RMHP.S19801

Jankowska-Polańska, P. B., Kuśnierz, M., Dudek, K., Jaroch, J. and Uchmanowicz, I. (2017). Impact of cognitive function on compliance with treatment in heart failure. Journal of Education, Health and Sport, 7(2), 392-414. https://doi.org/ 10.5281/zenodo.400294

Janssen, V., De Gucht, V., van Exel, H. and Maes, S. (2013). A self-regulation lifestyle program for post-cardiac rehabilitation patients has long-term effects on exercise adherence. Journal of Behavioral Medicine, 37(2), 308-321. https://doi.org/10.1007/s10865-012-9489-y

Janz, N. K. and Becker, M. H. (1984). The health belief model: A decade later. Health Education Quarterly, 11(1), 1-47. https://doi.org/10.1177/109019818401100101

Johnston, N., Bodegard, J., Jerström, S., Åkesson, J., Brorsson, H., Alfredsson, J., Karlsson, J.-E. and Varenhorst, C. (2016). Effects of interactive patient smartphone support app on drug adherence and lifestyle changes in myocardial infarction patients: A randomized study. American Heart Journal, 178, 85-94. https://doi.org/10.1016/j.ahj.2016.05. 005

Kähkönen, O., Saaranen, T., Kankkunen, P., Lamidi, M. L., Kyngäs, H. and Miettinen, H. (2018). Predictors of adherence to treatment by patients with coronary heart disease after percutaneous coronary intervention. Journal of Clinical Nursing, 27(5-6), 989-1003. https://doi.org/ 10.1111/jocn.14153

Kassavou, A. and Sutton, S. (2018). Automated telecommunication interventions to promote adherence to cardio-metabolic medications: Meta-analysis of effectiveness and meta-regression of behaviour change techniques. Health Psychology Review, 12(1), 25-42. https://doi.org/10.1080/17437199.2017.1365617

Kessing, D., Denollet, J., Widdershoven, J. and Kupper, N. (2016). Fatigue and self-care in patients with chronic heart failure. European Journal of Cardiovascular Nursing, 15(5), 337-344. https://doi.org/10.1177/1474515115575834
Kiecolt, K. J. (1994). Stress and the decision to change oneself: A theoretical model. Social Psychology Quarterly, 57(1), 49. https://doi.org/10.2307/2786974

King-Shier, K., Quan, H., Mather, C., Chong, E., LeBlanc, P. and Khan, N. (2018). Understanding ethno-cultural differences in cardiac medication adherence behavior: A Canadian study. Patient Prefer Adherence, 12, 1737-1747. https://doi.org/10.2147/PPA.S169167

Kolb, A. Y. and Kolb, D. A. (2009). The learning way: Metacognitive aspects of experiential learning. Simulation and Gaming, 40(3), 297-327. https://doi.org/10.1177/ 1046878108325713

Kosobucka, A., Michalski, P., Pietrzykowski, Ł., Kasprzak, M., Obońska, K., Fabiszak, T. and Kubica, A. (2018). Adherence to treatment assessed with the adherence in chronic diseases scale in patients after myocardial infarction. Patient Prefer Adherence, 12, 333-340. https://doi.org/ 10.2147/PPA.S150435

Lee, Y. M., Kim, R. B., Lee, H. J., Kim, K., et al. (2018). Relationships among medication adherence, lifestyle modification, and health-related quality of life in patients with acute myocardial infarction: A cross-sectional study. Health and Quality of Life Outcomes, 16(1), 100. https://doi.org/10.1186/s12955-018-0921-z

Leslie, K. H., McCowan, C. and Pell, J. P. (2019). Adherence to cardiovascular medication: A review of systematic reviews. Journal of Public Health, 41(1), e84-e94. https://doi.org/ 10.1093/pubmed/fdy088

Leung, A. W. Y., Chan, R. S. M., Sea, M. M. M. and Woo, J. (2017). An overview of factors associated with adherence to lifestyle modification programs for weight management in adults. International Journal of Environmental Research and Public Health, 14(8), 922. https://doi.org/10.3390/ ijerph14080922

Maeda, U., Shen, B. J., Schwarz, E. R., Farrell, K. A. and Mallon, S. (2013). Self-efficacy mediates the associations of social support and depression with treatment adherence in heart failure patients. International Journal of Behavioral Medicine, 20(1), 88-96. https://doi.org/10.1007/s12529011-9215-0

Marti, C., Georgiopoulou, V., Giamouzis, G., Cole, R. T., Deka, A., Tang, W. H. W. and Butler J. (2013). Patient-reported selective adherence to heart failure self-care recommendations: A prospective cohort study: The Atlanta Cardiomyopathy Consortium. Congestive Heart Failure, 19(1), 16-24. https://doi.org/10.1111/j.17517133.2012.00308.x

Martin, A. M. and Woods, C. B. (2012). What sustains longterm adherence to structured physical activity after a cardiac event? Journal of Aging and Physical Activity, 20(2), 135-147. https://doi.org/10.1123/japa.20.2.135

Martin, L. R., Williams, S. L., Haskard, K. B. and Dimatteo, M. R. (2005). The challenge of patient adherence. Therapeutics and Clinical Risk Management, 1(3), 189-199.

Mckenna, S. P. (1996). Predicting health behaviour: Research and practice with social cognition models. Safety Science, 24(3), 229-230. https://doi.org/10.1016/S09257535(97)814 $83-\mathrm{X}$ 
Metwaly, E. A. and Zatton, H. K. (2020). Effect of health educational program on self efficacy and therapeutic compliance among patients with myocardial infarction. Egyptian Journal of Health Care, 11(2), 214-228. https://doi.org710.21608/ejhc.2020.95786

Miller, N. H. (1997). Compliance with treatment regimens in chronic asymptomatic diseases. American Journal of Medicine, 102(2A), 43-49. https://doi.org/10.1016/s00029343(97)00467-1

Muka, T., Koromani, F., Portilla, E., O’Connor, A., Bramer, W. M., Troup, J. and Franco, O. H. (2016). The role of epigenetic modifications in cardiovascular disease: A systematic review. International Journal of Cardiology, 212, 174-183. https://doi.org/10.1016/j.ijcard.2016.03.062

Murray, J., Craigs, C. L., Hill, K. M., Honey, S. and House, A. (2012). A systematic review of patient reported factors associated with uptake and completion of cardiovascular lifestyle behaviour change. BMC Cardiovascular Disorders, 12, 120. https://doi.org/10.1186/1471-2261-12-120

Nieuwenhuis, M. M., Jaarsma, T., van Veldhuisen, D. J., Postmus, D. and van der Wal, M. H. (2012). Long-term compliance with nonpharmacologic treatment of patients with heart failure. American Journal of Cardiology, 110(3), 392-397. https://doi.org/10.1016/j.amjcard.2012.03.039

O'Grady, L., Witteman, H. and Wathen, C. (2008). The experiential health information processing model: Supporting collaborative web-based patient education. BMC Medical Informatics and Decision Making, 8, 58. https://doi.org/10.1186/1472-6947-8-58

Ok, J. S. and Choi, H. (2015). Factors affecting adherence to self-care behaviors among outpatients with heart failure in Korea. Korean Journal of Adult Nursing, 27(2), 242. https://doi.org/10.7475/kjan.2015.27.2.242

Olshansky B. (2007). Placebo and nocebo in cardiovascular health: implications for healthcare, research, and the doctor-patient relationship. Journal of the American College of Cardiology, 49(4), 415-421. https://doi.org/10.1016/ j.jacc.2006.09.036

Oosterom-Calo, R., van Ballegooijen, A., Terwee, C., te Velde, S. J., Brouwer, I. A., Jaarsma, T. and Brug, J. (2012). Determinants of adherence to heart failure medication: A systematic literature review. Heart Failure Review, 18(4), 409-427. https://doi.org/10.1007/s10741-012-9321-3

Pepitone, A. and Festinger, L. (1959). A theory of cognitive dissonance. American Journal of Psychology, 72, 153. https://doi.org/10.2307/1420234

Pfaeffli Dale, L., Dobson, R., Whittaker, R. and Maddison, R. (2016). The effectiveness of mobile-health behaviour change interventions for cardiovascular disease selfmanagement: A systematic review. European Journal of Preventive Cardiology, 23(8), 801-817. https://doi.org/ $10.1177 / 2047487315613462$
Piotrowicz, E., Zieliński, T., Bodalski, R., Rywik, T., Dobraszkiewicz-Wasilewska, B., Sobieszczańska-Małek, M. and Piotrowicz, R. (2015). Home-based tele monitored Nordic walking training is well accepted, safe, effective and has high adherence among heart failure patients, including those with cardiovascular implantable electronic devices: A randomised controlled study. European Journal of Preventive Cardiology, 22(11), 1368-1377. https://doi.org/ $10.1177 / 2047487314551537$

Prochaska, J. O. and DiClemente, C. C. (2005). The transtheoretical approach. In J. C. Norcross and M. R. Goldfried (Eds.), Handbook of psychotherapy integration. Oxford series in clinical psychology (pp. 147-171). Oxford; New York: Oxford University Press.

Rashidi, A., Kaistha, P., Whitehead, L. and Robinson, S. (2020). Factors that influence adherence to treatment plans amongst people living with cardiovascular disease: A review of published qualitative research studies. International Journal of Nursing Studies, 110, 103727. https://doi.org/10.1016/j.ijnurstu.2020.103727

Rich, A., Brandes, K., Mullan, B. and Hagger, M. S. (2015). Theory of planned behavior and adherence in chronic illness: A meta-analysis. Journal of Behavioral Medicine, 38(4), 673-688. https://doi.org/10.1007/s10865-015-96443

Rosenstock, I. (1974). Historical origins of the health belief model. Health Education \& Behavior, 2(4), 328-335. https://doi.org/10.1177/109019817400200403

Sabaté, E. and Cluster, W. (2001). Adherence to long-term therapies: Policy for action: Meeting report. Available at: https://apps.who.int/iris/handle/10665/66984

Santo, K., Hyun, K., de Keizer, L., Thiagalingam, A., Hillis, G. S., Chalmers, J., Redfern, J. and Chow, C. K. (2018). The effects of a lifestyle-focused text-messaging intervention on adherence to dietary guideline recommendations in patients with coronary heart disease: An analysis of the TEXT ME study. International Journal of Behavioral Nutrition and Physical Activity, 15, 45. https://doi.org/ 10.1186/s12966-018-0677-1

Seid, M. A., Abdela, O. A. and Zeleke, E. G. (2019). Adherence to self-care recommendations and associated factors among adult heart failure patients. From the patients' point of view. PLoS One, 14(2), e0211768. https://doi.org/ 10.1371/journal.pone.0211768

Serour, M., Alqhenaei, H., Al-Saqabi, S., Mustafa, A. R. and Ben-Nakhi, A. (2007). Cultural factors and patients' adherence to lifestyle measures. British Journal of General Practice, 57(537), 291-295.

Shan, Z., Li, Y., Zong, G., Guo, Y., Li, J., Manson, J. E. and Bhupathiraju, S. N. (2018). Rotating night shift work and adherence to unhealthy lifestyle in predicting risk of type 2 diabetes: Results from two large US cohorts of female nurses. BMJ, 363, k4641.https://doi.org/10.1136/bmj.k4641 
Slovinec D’Angelo, M. E., Pelletier, L. G., Reid, R. D. and Huta, V. (2014). The roles of self-efficacy and motivation in the prediction of short- and long-term adherence to exercise among patients with coronary heart disease. Health Psychology, 33(11), 1344-1353. https://doi.org/10.1037/ hea0000094

Sol, B. G., van der Graaf, Y., van Petersen, R. and Visseren, F. L. (2011). The effect of self-efficacy on cardiovascular lifestyle. European Journal of Cardiovascular Nursing, 10(3), 180-186. https://doi.org/10.1016/j.ejcnurse.2010.06.005

Stonerock, G. L. and Blumenthal, J. A. (2017). Role of counseling to promote adherence in healthy lifestyle medicine: Strategies to improve exercise adherence and enhance physical activity. Progress in Cardiovascular Diseases, 59(5), 455-462. https://doi.org/10.1016/j.pcad. 2016.09.003

Suhail, M., Saeed, H., Saleem, Z., Younas, S., Hashmi, F. K., Rasool, F. and Imran, I. (2021). Association of health literacy and medication adherence with health-related quality of life (HRQoL) in patients with ischemic heart disease. Health and Quality of Life Outcomes, 19(1), 118. https://doi.org/10.1186/s12955-021-01761-5

Taher, M., Safavi Bayat, Z., Niromand Zandi, K., Ghasemi, E., Abredari, H., Karimy, M. and Abedi, A. R. (2015). Correlation between compliance regimens with health locus of control in patients with hypertension. Medical Journal of the Islamic Republic of Iran, 29, 194.

Tawalbeh, L. I. and Ahmad, M. M. (2014). The effect of cardiac education on knowledge and adherence to healthy lifestyle. Clinical Nursing Research, 23(3), 245-258. https://doi.org/10.1177/1054773813486476

Tawalbeh, L. I., Al Qadire, M., Ahmad, M. M., Aloush, S., Abu Sumaqa, Y. and Halabi, M. (2017). Knowledge and self-care behaviors among patients with heart failure in Jordan. Research in Nursing and Health, 40(4), 350-359. https://doi.org/10.1002/nur.21805

Thornton, C. S., Tsai, W. H., Santana, M. J., Penz, E. D., Flemons, W. W., Fraser, K. L. and Pendharkar, S. R. (2020). Effects of wait times on treatment adherence and clinical outcomes in patients with severe sleep-disordered breathing: A secondary analysis of a non-inferiority randomized clinical trial. JAMA Network Open, 3(4), e203088. https://doi.org/10.1001/jamanetworkopen.2020. 3088

Tierney, S., Mamas, M., Woods, S., Rutter, M. K., Gibson, M., Neyses, L. and Deaton, C. (2012). What strategies are effective for exercise adherence in heart failure? A systematic review of controlled studies. Heart Failure Reviews, 17(1), 107-115. https://doi.org/10.1007/s10741011-9252-4
Tilson, H. H. (2004). Adherence or compliance? Changes in terminology. Annals of Pharmacotherapy, 38(1), 161-162. https://doi.org/10.1345/aph.1D207

Unverzagt, S., Meyer, G., Mittmann, S., Samos, F. A., Unverzagt, M. and Prondzinsky, R. (2016). Improving treatment adherence in heart failure. Deutsches Arzteblatt International, 113(25), 423-430. https://doi.org/10.3238/ arztebl.2016.0423

Urbinati, S., Olivari, Z., Gonzini, L., Savonitto, S., Farina, R., Del Pinto, M. and BLITZ-4 Investigators. (2015). Secondary prevention after acute myocardial infarction: Drug adherence, treatment goals, and predictors of health lifestyle habits. The BLITZ-4 registry. European Journal of Preventive Cardiology, 22(12), 1548-1556. https://doi.org/ $10.1177 / 2047487314561876$

van der Laan, D. M., Elders, P. J. M., Boons, C. C. L. M., Nijpels, G. and Hugtenburg, J. G. (2019). Factors associated with nonadherence to cardiovascular medications: A crosssectional study. Journal of Cardiovascular Nursing, 34(4), 344-352. https://doi.org/10.1097/JCN.0000000000000582

van der Wal, M. H., van Veldhuisen, D. J., Veeger, N. J., Rutten, F. H. and Jaarsma, T. (2010). Compliance with nonpharmacological recommendations and outcome in heart failure patients. European Heart Journal, 31(12), 1486-1493. https://doi.org/10.1093/eurheartj/ehq091

Wallert, J., Gustafson, E., Held, C., Madison, G., Norlund, F., von Essen, L. and Olsson, E. M. G. (2018). Predicting adherence to internet-delivered psychotherapy for symptoms of depression and anxiety after myocardial infarction: Machine learning insights from the U-C A R E Heart randomized controlled trial. Journal of Medical Internet Research, 20(10), e10754. https://doi.org/10.2196/ 10754

Westland, H., Jaarsma, T., Riegel, B., Iovino, P., Henry Osokpo, O., Stawnychy, M. and Tarbi, E. (2020). Self-care interventions in patients with coronary artery disease: Room for improvement. European Heart Journal, 41, ehaa946.3412. https://doi.org/10.1093/ehjci/ehaa946.3412

World Health Organization. (2003). Adherence to long-term therapies: Evidence for action. Available at: http://apps.who.int/iris/bitstream/handle/10665/42682/92 41545992.pdf? sequence $=1$

World Health Organization. (2020). WHO reveals leading causes of death and disability worldwide: 2000-2019. Available at: https://www.who.int/news/item/09-12-2020who-reveals-leading-causes-of-death-and-disabilityworldwide-2000-2019

Yu, D. S., Lee, D. T. and Woo, J. (2010). Improving healthrelated quality of life of patients with chronic heart failure: Effects of relaxation therapy. Journal of Advanced Nursing, 66(2), 392-403. https://doi.org/10.1111/j.1365-2648. 2009.05198.x 